\title{
Sputum bacteriology conversion and treatment outcome of patients with multidrug-resistant tuberculosis
}

This article was published in the following Dove Press journal: Infection and Drug Resistance

\author{
Lingshuang $L v^{1, *}$ \\ Tiecheng $\mathrm{Li}^{2, *}$ \\ Kun $\mathrm{Xu}{ }^{\prime}$ \\ Peiyi Shi' \\ Biyu $\mathrm{He}^{\prime}$ \\ Weimin Kong ${ }^{3}$ \\ Jianming Wang' \\ Jian Sun ${ }^{3}$
}

'Department of Epidemiology, Key Laboratory of Infectious Diseases, School of Public Health, Nanjing Medical University, Nanjing, China; ${ }^{2}$ Department of Tuberculosis, the Fourth People's Hospital of Lianyungang City, Lianyungang, China; ${ }^{3}$ Department of Thoracic Surgery, the First People's Hospital of Yancheng City, Yancheng, China

*These authors contributed equally to this work
Correspondence: Jianming Wang Department of Epidemiology, Key Laboratory of Infectious Diseases, School of Public Health, Nanjing Medical University, I0I Longmian Avenue, Jiangning District, Nanjing, 21 I I66, China Tel +862586868438

Email jmwang@njmu.edu.cn

Jian Sun

Department of Thoracic Surgery, the First People's Hospital of Yancheng City, 166 Yulong West Road, Tinghu District, Yancheng, 22400I, China Email I5895I91698@I63.com
Purpose: Multidrug-resistant tuberculosis (MDR-TB) requires long-term treatment, has a high fatality rate, and constitutes a global threat. Earlier detection of treatment failure is required to predict therapeutic efficacy.

Patients and methods: We enrolled MDR-TB patients consecutively from January 2011 through December 2012 in Lianyungang, China. Sputum smear microscopy tests and sputum cultures were performed once a month for the first 6 months following initiation of antituberculosis treatment and once every 2 months thereafter until the end of therapy. The sensitivity, specificity and area under the receiver operating characteristic curve (AUC) were used with a 95\% CI to estimate the role of sputum bacteriology conversion in predicting treatment outcomes. Results: Among the 92 MDR-TB patients enrolled in this study, 40.2\% had poor treatment outcomes. The median initial sputum bacteriology conversion time was 1 month. Patients having 2-month sputum smear conversions (adjusted odds ratio [OR]: 7.19, 95\% CI: 2.60-19.84) or culture conversions (adjusted OR: 2.88, 95\% CI: 1.11-7.45) were more likely to experience good outcomes. The sensitivity and specificity obtained when using two-month sputum smear conversions to predict treatment outcomes were $67.6 \%$ (95\% CI: $50.2-82.0)$ and $76.4 \%$ (95\% CI: $63.0-86.8$ ), respectively. The sensitivity and specificity obtained when using 2-month culture conversions to predict treatment outcomes were $48.6 \%$ (95\% CI: $32.0-65.6)$ and $74.5 \%$ (95\% CI: 61.0-85.3), respectively. The AUC for two-month smear conversions was $0.72(95 \% \mathrm{CI}$ : $0.62-0.81)$, significantly higher than that obtained for 2 -month culture conversions $(0.62,95 \%$ CI: $0.52-0.72)\left(\chi^{2}=4.18, P=0.041\right)$.

Conclusion: The prognoses of MDR-TB patients displaying persistent sputum positivity were inferior to those for whom sputum bacteriology conversion was observed. Thus, sputum smear conversion results obtained 2 months after treatment initiation may provide a potential means for predicting MDR-TB treatment outcomes.

Keywords: multidrug-resistant tuberculosis, sputum smear conversion, sputum culture conversion, treatment outcome, prognosis

\section{Introduction}

Tuberculosis (TB) is a chronic infectious disease caused by the Mycobacterium tuberculosis complex. Although the occurrence of TB worldwide has slowly declined in recent years (by a rate of $\sim 1.5 \%$ per year), the disease burden remains remarkably heavy in developing countries. ${ }^{1}$ The highly cost-effective DOTS (direct observed therapy, with short course) strategy has helped to bring this global epidemic under control in many parts of the world; however, the emergence and spread of drug-resistant strains pose a major threat to these achievements. ${ }^{2}$ In 2014, approximately 9.6 million TB cases and 
1.5 million TB deaths were reported worldwide, with 3.3\% of these cases resulting from multidrug-resistant (MDR) or extensively drug-resistant (XDR) strains. ${ }^{3}$ The status of drugresistant TB in China is notably grim and exhibits regional epidemiologic characteristics. ${ }^{4,5}$

MDR-TB, which is caused by infection with M. tuberculosis, is resistant to isoniazid (INH) and rifampicin (RIF) at least, ${ }^{6}$ further reducing the chances of successful TB treatment and making it more difficult. The extent to which drug resistance affects the outcomes of anti-TB treatment depends on patterns of drug resistance, treatment regimens, treatment adherence and each patient's immunity. ${ }^{3}$ The recommended regimen for MDR-TB is toxic, poorly tolerated, prolonged (up to 24 months), and not evidence-based (i.e., based on data obtained from randomized controlled clinical trials). ${ }^{8}$ The core drugs used to treat MDR-TB are fluoroquinolones (FQ), such as moxifloxacin (MXF), levofloxacin (LVX) or ofloxacin (OFX), and second-line injectable agents like kanamycin (KM), amikacin (AMK) or capreomycin (CPM). One or more oral second-line drugs are required to build an effective MDR-TB regimen, and the adverse effects are challenges. ${ }^{3}$

The success rate of treating MDR-TB varies between countries but remains low. ${ }^{6}$ In a 2012 cohort, the overall proportion of completed treatments (i.e., cures or treatments completed) was $50 \%$, with the highest and lowest rates occurring in the eastern Mediterranean region (65\%) and the European and Southeast Asia regions (49\%), respectively. ${ }^{3}$ The emergence of incurable TB can result in community-based transmission of untreatable strains, which has raised legal, ethical, and logistical dilemmas regarding patient placement and rights to unrestricted travel and work. ${ }^{7}$

Sputum smear microscopy and, ideally, sputum cultures are recommended for monitoring the effectiveness of antiTB chemotherapy, where sputum smear is available, and culture is ideal. The sputum smear microscopy tests, taken two months after treatment is initiated, are recommended for low-resource settings that lack the ability to perform culture or drug susceptibility testing (DST). ${ }^{8}$ Sputum culture conversion has been demonstrated by clinical doctors to be useful for predicting the therapeutic efficacy of TB treatment, while sputum bacteriology conversion has been reported to be a useful indicator of MDR-TB treatment outcomes. ${ }^{7,9}$ These results suggest that sputum conversion at 6 months may predict treatment success with higher sensitivity. However, the subjects involved in this study were recruited from multiple sites over a short time, which may have resulted in selection bias. Moreover, by the time one waits 6 months to predict the risk of treatment failure, it may be too late for the patient to benefit from changes in the treatment regimen. In order to further explore the potential of this early marker for predicting MDR-TB treatment outcomes, we obtained the subjects of the present study by continuously recruiting MDR-TB patients from one city in China and followed them, using a cohort study design, through their outcomes.

\section{Patients and methods Study subjects}

Our analysis included all new and previously treated MDRTB patients identified from January 2011 to December 2012 in the Fourth People's Hospital of Lianyungang City, China. These patients were diagnosed with pulmonary MDR-TB based on the results of DSTs on Lowenstein-Jensen culture media. Figure 1 shows the flowchart of patient recruitment. Two standard protocols (SR1 and SR2) were used to treat MDR-TB patients. SR1 represents $6 Z$ Am Lfx PAS Pto/18Z Lfx PAS Pto, while SR2 represents $6 \mathrm{Z}$ Am Lfx EMB Pto/18Z Lfx EMB Pto. Both sputum mycobacterial cultures and sputum smear microscopy tests were performed once a month for the first 6 months and once every 2 months from that point until the end of therapy. At enrollment, the subjects completed a structured questionnaire that enabled the determination of demographic characteristics (gender, date of birth, occupation, etc.), behaviors (tobacco smoking, alcohol drinking, etc.), previous anti-TB treatment history and baseline DST results. Patients were followed for their treatment adherence and outcomes. The study protocol was approved by the Institutional Review Board of Nanjing Medical University. Written informed consent was obtained from all study participants.

\section{Definitions}

We mainly measured the following four types of bacteria conversion: initial sputum smear conversion, initial sputum culture conversion, 2-month sputum smear conversion, and 2-month culture conversion. The "time to sputum conversion" was defined as the time in days from the initiation of MDRTB treatment to the date on which the first of consecutive negative sputum conditions was detected. ${ }^{10}$ For patients who never converted, whether due to treatment interruption or failure to follow-up, the "time to initial conversion" was the number of days from the initiation of MDR-TB treatment to their last sputum smear and culture detection. A 2-month sputum smear/culture conversion pertained when two consecutive negative sputum condition examinations were obtained during the first 2 months. Initial sputum bacteriology 


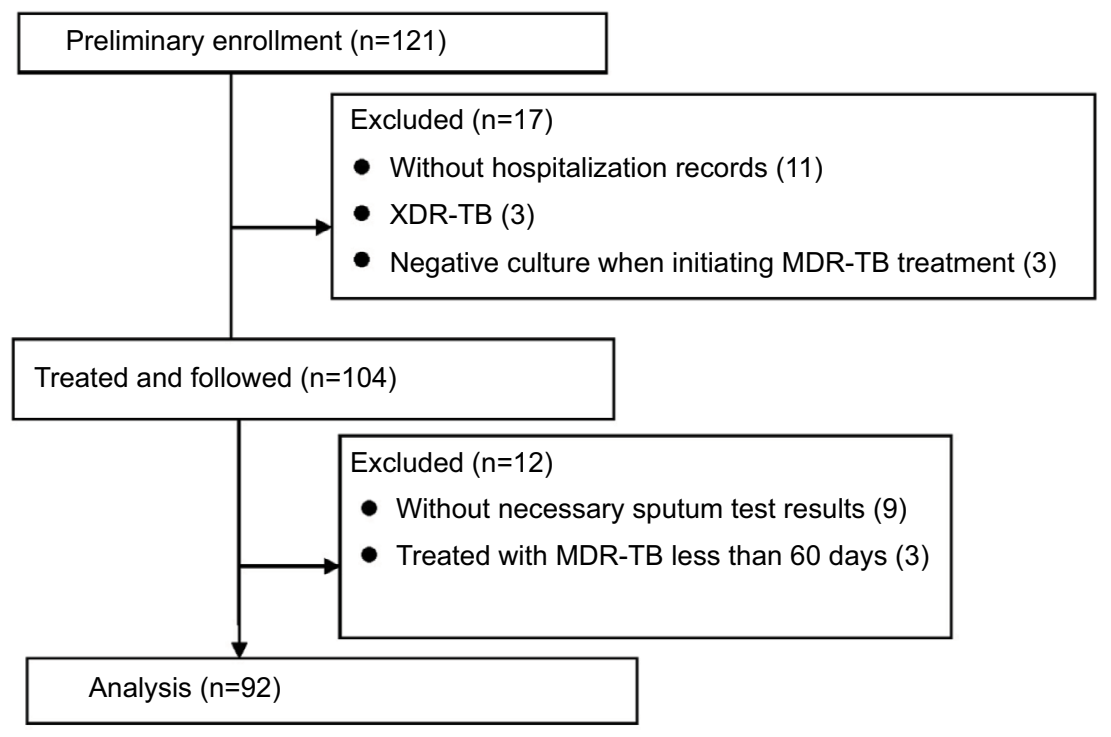

Figure I Flowchart of patient recruitment.

Abbreviations: TB, tuberculosis; MDR-TB, multidrug-resistant TB; XDR-TB, extensively drug-resistant TB.

conversion pertained when at least two consecutive negative sputum specimens were collected at least 1 month apart. We also measured the sputum bacteria status at the end of 3- or 6-month periods in relation to treatment outcomes.

Treatment outcomes were defined as follows, according to WHO (World Health Organization) and IUATLD (International Union against Tuberculosis and Lung Disease) guidelines: ${ }^{11}$ (1) cured (finished treatment with a negative bacteriology result at the end of treatment); (2) treatment failure (remaining smear positive at five months despite correct intake of medication); (3) treatment completed (treatment was finished but without obtaining bacteriology results at the end of the treatment periods for smear-positive subjects); (4) death (patients died during the course of treatment); (5) defaulter (patients who interrupted their treatment for 2 consecutive months or more after registration); and (6) transfer-out (patients whose treatment results were unknown due to transfer to another health facility). Categories (1) and (3) were classified as good outcomes. Categories (2), (4), and (5) were classified as poor outcomes.

\section{Data analysis}

Data were entered, cleaned, and analyzed using the statistical package SPSS 18.0 (IBM Corporation, New York, USA). Frequencies and proportions were used to describe patient characteristics and treatment outcomes. The Pearson chisquare or likelihood chi-square test was used to compare frequencies between groups. For survival data, the time to initial sputum smear/culture conversion was analyzed using the Kaplan-Meier test. The log-rank test was used to explore the related factors for initial smear/culture conversion. To estimate the strength of the relation between bacteriology status and a good treatment outcome, we calculated the OR and $95 \% \mathrm{CI}$ using the logistic regression model. The sensitivity and specificity were used to estimate the effectiveness of sputum bacteriology conversion in predicting treatment outcomes. Sensitivity was defined as the proportion of patients with sputum smear/culture conversion among those with good treatment outcomes. Specificity was defined as the proportion of patients without sputum smear/culture conversion among those with poor treatment outcomes. ${ }^{12}$ The receiver operating characteristic (ROC) curve was plotted to visualize the effect of sputum bacteriology conversion on the balance between sensitivity and specificity. A $P$-value of $<0.05$ was considered significant.

\section{Results \\ General characteristics}

Out of the 121 patients available at the preliminary enrollment, 92 (men: 59; women: 33) met the inclusive criteria and were included in our analysis (Figure 1). The mean age was 46.5 (range: 18-79) years. There was no significant difference in age between male (49.0 years) and female patients (42.2 years) $(t=-1.928, P=0.057)$. Among the $92 \mathrm{MDR}-\mathrm{TB}$ patients enrolled in this study, $40.2 \%$ (37/92) had poor treatment outcomes, including defaulter $(25.0 \%, 23 / 92)$, treatment failure $(12.0 \%, 11 / 92)$ and death $(3.3 \%, 3 / 92)$. Patients who exhibited drug resistance to OFX $\left(\chi^{2}=9.40, P=0.002\right)$ or who were $\geq 60$ years old $\left(\chi^{2}=4.16, P=0.041\right)$ were more likely to have poor outcomes (Table 1). 
Table I Demographic and clinical characteristics of the study population $(n=92)$

\begin{tabular}{|c|c|c|c|c|c|}
\hline \multirow[t]{2}{*}{ Characteristics } & \multirow{2}{*}{$\begin{array}{l}\text { Cases, } \\
\text { n (\%)* }\end{array}$} & \multicolumn{2}{|c|}{ Treatment outcomes } & \multirow[t]{2}{*}{$\chi^{2}$} & \multirow[t]{2}{*}{$P$} \\
\hline & & Good, n (\%) & Poor, n (\%) & & \\
\hline Age, years & & & & 4.16 & 0.041 \\
\hline$<60$ & $72(78.3)$ & $47(85.5)$ & $25(67.6)$ & & \\
\hline$\geq 60$ & $20(21.7)$ & $8(14.5)$ & $12(32.4)$ & & \\
\hline Gender & & & & 0.32 & 0.570 \\
\hline Male & $33(35.9)$ & $21(38.2)$ & $12(32.4)$ & & \\
\hline Female & $59(64.1)$ & $34(61.8)$ & $25(67.6)$ & & \\
\hline Occupation & & & & 0.64 & 0.424 \\
\hline Farmer & $68(73.9)$ & 39 (70.9) & $29(78.4)$ & & \\
\hline Non-farmer & $24(26.1)$ & $16(29.1)$ & $8(21.6)$ & & \\
\hline Smoking & & & & 0.44 & 0.509 \\
\hline No & $61(66.3)$ & $35(63.6)$ & $26(70.3)$ & & \\
\hline Yes & $31(33.7)$ & $20(36.4)$ & II (29.7) & & \\
\hline Alcohol use & & & & 1.33 & 0.249 \\
\hline No & $80(87.0)$ & $46(83.6)$ & $34(91.9)$ & & \\
\hline Yes & $12(13.0)$ & $9(16.4)$ & $3(8.1)$ & & \\
\hline Treatment regimens & & & & 0.01 & 0.903 \\
\hline SRI & $38(4 I .3)$ & $23(4 I .8)$ & $15(40.5)$ & & \\
\hline SR2 & $54(58.7)$ & $32(58.2)$ & $22(59.5)$ & & \\
\hline Previous treatment history & & & & 0.32 & 0.852 \\
\hline No & $33(35.9)$ & $21(38.2)$ & $12(32.4)$ & & \\
\hline First-line drugs & $21(22.8)$ & $12(21.8)$ & $9(24.3)$ & & \\
\hline First- and second-line drugs & $38(4 \mid .3)$ & $22(40.0)$ & $16(43.2)$ & & \\
\hline Resistance to OFX & & & & 9.40 & 0.002 \\
\hline Yes & $22(23.9)$ & $7(12.7)$ & $15(40.5)$ & & \\
\hline No & $70(76.1)$ & $48(87.3)$ & $22(59.5)$ & & \\
\hline Resistance to KM & & & & -- & -- \\
\hline Yes & $92(100)$ & $55(59.8)$ & $37(40.2)$ & & \\
\hline No & 0 & 0 & 0 & & \\
\hline Resistance to EMB & & & & 0.25 & 0.616 \\
\hline Yes & $24(35.8)$ & $13(33.3)$ & II (39.3) & & \\
\hline No & $43(64.2)$ & $26(66.6)$ & $17(60.7)$ & & \\
\hline Resistance to SM & & & & 0.36 & 0.549 \\
\hline Yes & $34(50.7)$ & $21(53.8)$ & $13(46.4)$ & & \\
\hline No & $33(49.3)$ & $18(46.2)$ & $15(53.6)$ & & \\
\hline
\end{tabular}

Notes: *Not all cases were tested for drug resistance to EMB and SM.

Abbreviations: EMB, ethambutol; KM, kanamycin; OFX, ofloxacin; SM, streptomycin.

\section{Initial sputum bacteriology conversion and treatment outcome}

Initial sputum bacteriology (both smear and culture) conversions were observed in $87.0 \%$ (80/92) of the patients in this study. The median conversion time for these patients was 1 month, and 13.0\% (12/92) did not convert. Patients exhibiting drug resistance to ethambutol (EMB) or OFX were less likely to exhibit an initial sputum bacteriology conversion (Table 2). Patients with good treatment outcomes exhibited significantly shorter times to initial sputum bacteriology conversion (median: 1 month) than those with poor treatment outcomes (median: 2 months) $(P<0.05)$. The chances of a poor treatment outcome were significantly associated with both the positive status of the remaining sputum smear
(Figure 2A) and the positive status of the sputum culture (Figure 2B). Patients exhibiting initial sputum smear conversion and culture conversion had a $66.3 \%(53 / 80)$ probability of good treatment outcomes; however, their proportion was only $16.7 \%$ (2/12) among those who did not exhibit sputum bacteriology conversion. Patients exhibiting initial sputum smears or culture conversions experienced more good treatment outcomes than those who did not exhibit conversion $\left(\chi^{2}=9.11, P=0.003\right.$ and $\chi^{2}=4.87, P=0.027$, respectively).

\section{Two-month sputum bacteriology conversion and treatment outcome}

In the first 2 months of treatment, the proportions of subjects exhibiting sputum smear conversions and culture conversions 
Table 2 Drug resistance to ofloxacin and ethambutol associated with initial sputum bacteriology conversions among patients treated for MDR-TB

\begin{tabular}{|c|c|c|c|c|c|c|c|c|}
\hline \multirow[t]{2}{*}{ Characteristics } & \multicolumn{4}{|c|}{ Initial smear conversion } & \multicolumn{4}{|c|}{ Initial culture conversion } \\
\hline & $n$ & Converted & $\chi^{2 \#}$ & $P^{\#}$ & $\mathbf{n}$ & Converted & $\chi^{2 \#}$ & $P^{\#}$ \\
\hline Resistance to OFX & & & 4.04 & 0.044 & & & 3.05 & 0.081 \\
\hline Yes & 22 & 17 & & & 22 & 17 & & \\
\hline No & 70 & 63 & & & 70 & 63 & & \\
\hline Resistance to EMB* & & & 5.32 & 0.021 & & & 7.92 & 0.005 \\
\hline Yes & 24 & 20 & & & 24 & 20 & & \\
\hline No & 43 & 42 & & & 43 & 42 & & \\
\hline
\end{tabular}

Notes: *67 cases were tested for drug resistance to EMB; \#Log-rank test.

Abbreviations: EMB, ethambutol; MDR-TB, multidrug-resistant tuberculosis; OFX, ofloxacin.

A

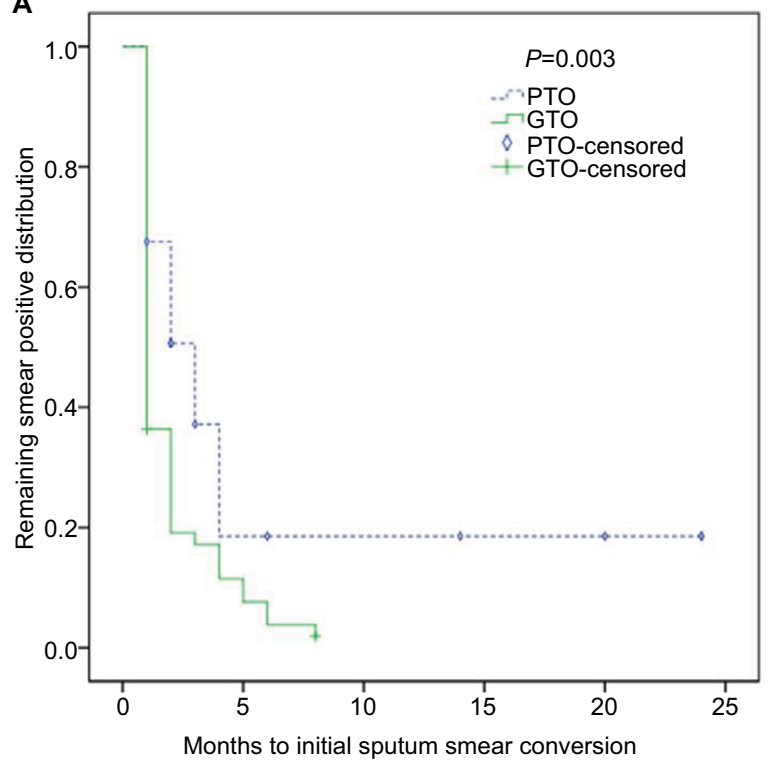

B

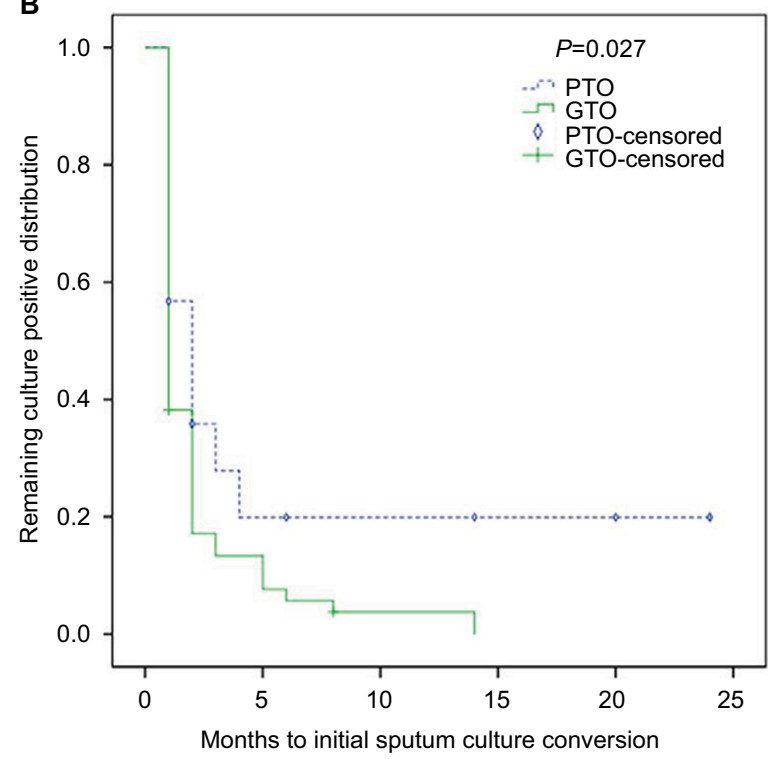

C

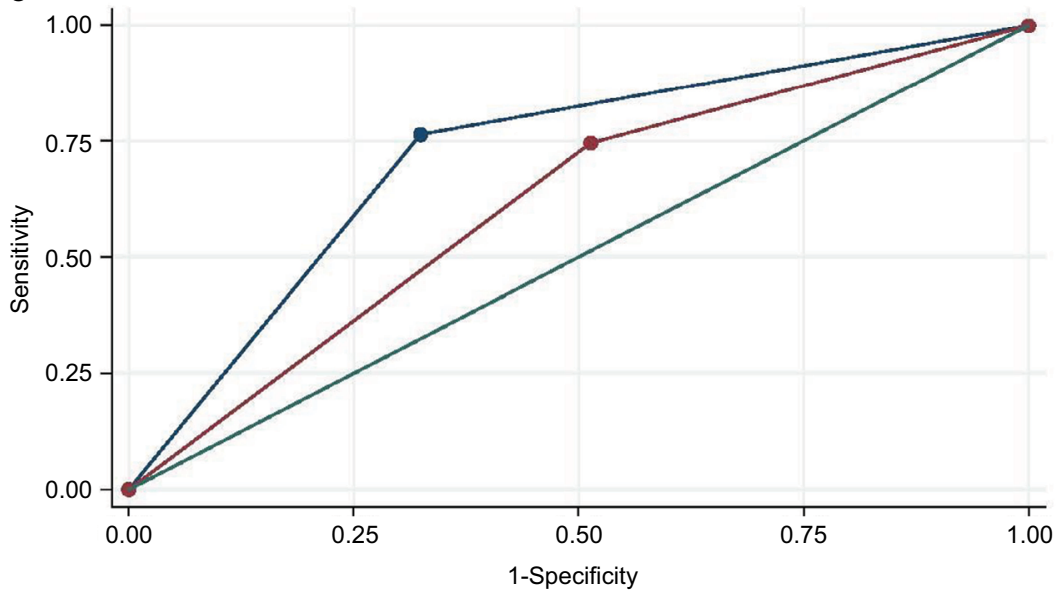

$\longrightarrow$ Two-month smear conversion AUC=0.72 (95\% Cl: 0.62-0.81)

$\longrightarrow$ Two-month culture conversion AUC=0.62 (95\% Cl: 0.52-0.72)

Reference

Figure 2 Sputum bacteriology conversions and treatment outcomes. (A) Initial sputum smear conversions and treatment outcomes; (B) initial sputum culture conversions and treatment outcomes; (C) diagnostic performance of 2-month sputum bacteriology conversions in predicting treatment outcomes.

Abbreviations: AUC, area under the receiver operating characteristic curve; GTO, good treatment outcome; PTO, poor treatment outcome. 
were 58.7\% (54/92) and 64.1\% (59/92), respectively. Patients exhibiting 2-month sputum smear conversions (adjusted OR: 7.19, 95\% CI: 2.60-19.84) or culture conversions (adjusted OR: $2.88,95 \%$ CI: $1.11-7.45)$ were more likely to experience good outcomes (Table 3). Using 2-month sputum smear conversions to predict treatment outcomes resulted in a sensitivity and specificity of $67.6 \%$ (95\% CI: 50.2-82.0) and $76.4 \%$ (95\% CI: 63.0-86.8), respectively. Using 2-month sputum culture conversions to predict treatment outcomes resulted in a sensitivity and specificity of $48.6 \%$ (95\% CI: $32.0-65.6)$ and $74.5 \%$ (95\% CI: 61.0-85.3), respectively. The area under the receiver operating characteristic curve (AUC) for 2-month sputum smear conversions was 0.72 (95\% CI: 0.62-0.81), significantly higher than the AUC for the 2-month sputum culture conversions $(0.62,95 \%$ CI: $0.52-0.72)\left(\chi^{2}=4.18\right.$, $P=0.041$ ) (Figure 2C).

\section{Three- or six-month sputum bacteriology status and treatment outcome}

We further explored the ability to predict treatment outcomes using the sputum bacteriology status obtained at the third or sixth month. The AUC values obtained using the third- and sixth-month smear status were 0.67 (95\% CI: 0.58-0.77) and 0.62 (95\% CI: 0.52-0.71), respectively. The AUC values obtained using the 3rd- and 6th-month culture status were 0.63 (95\% CI: $0.53-0.71)$ and 0.65 (95\% CI: $0.56-0.74)$, respectively.

\section{Discussion}

The retrospective cohort study contained herein explores the potential of using a patient's sputum bacteriology status to predict his/her MDR-TB treatment outcome. Approximately $60 \%$ of the MDR-TB patients included in this study achieved sputum smear or culture conversions at the end of 2 months of treatment, and more than $85.0 \%$ of the study subjects achieved initial sputum bacteriology conversions. The proportion of patients who experienced good treatment outcomes was near $60 \%$, which is consistent with the average success rate but still below the target goal of $75.0 \% .{ }^{13} \mathrm{We}$ observed that MDR-TB patients exhibiting persistent sputum positivity had worse prognoses than those exhibiting sputum bacteriology conversions. Thus, the occurrence of sputum smear conversion at the end of 2 months of treatment may be a useful marker for predicting a patient's MDR-TB treatment outcome.

MDR-TB treatment is prolonged, expensive, poisonous, complicated, and often unsuccessful. ${ }^{14}$ The regimens vary widely due to differences in opinions as well as available resources. MDR-TB patients who received individualized regimens have experienced a higher success rate than patients who received standardized regimens (64\% versus $62 \%$, respectively, $P<0.001) .{ }^{15}$ In our study, we found that patients sensitive to OFX were more likely to experience good treatment outcomes, indicating that patients exhibiting specific characteristics may be more likely to experience successful treatment outcomes.

Clinical, radiological and immunological features have shown potential as predictors of TB treatment outcomes, but the reported studies lack validity and reliability. ${ }^{16}$ Studies have demonstrated that monitoring drug concentration and bacteriology is essential for predicting treatment response. ${ }^{17,18}$ Sputum bacteriology conversion has been the most widely used surrogate marker for defining TB treatment outcomes. ${ }^{19,20}$ Lu et al recommended using the sputum culture conversion status at the 6th month to predict the success of MDR-TB treatment. ${ }^{9}$ Kurbatova et al suggested that culture conversion status at the 9th month, with five consecutive negative cultures conversion, may more accurately predict a patient's MDR-TB prognosis. ${ }^{21}$ However, the 6 or 9 months thereby required to predict MDR-TB treatment outcomes may mean that the results come too late. Identifying an early predictor is crucial if treatment regimens are to be changed in time to be effective. In our study, we observed that the sputum bacteriology conversion occurring at 2 months demonstrated superior predictive value. Considering the lack of availability of cultures in resource-limited settings, the sputum smear

Table 3 Association of 2-month sputum bacteriology conversions with treatment outcomes

\begin{tabular}{|c|c|c|c|c|c|}
\hline \multirow[t]{2}{*}{ Characteristics } & \multirow{2}{*}{$\begin{array}{l}\text { Case } \\
\text { n (\%) }\end{array}$} & \multicolumn{2}{|c|}{ Treatment outcome } & \multirow[t]{2}{*}{$P^{*}$} & \multirow[t]{2}{*}{ OR $(95 \% \mathrm{Cl}) *$} \\
\hline & & Good, n (\%) & Poor, n (\%) & & \\
\hline Two-month smear conversion & & & & $<0.001$ & $7.19(2.60-19.84)$ \\
\hline Yes & $54(58.7)$ & $42(76.4)$ & $12(32.4)$ & & \\
\hline No & $38(4 \mid .3)$ & $13(23.6)$ & $25(67.6)$ & & \\
\hline \multicolumn{6}{|l|}{ Two-month culture conversion } \\
\hline Yes & $60(65.2)$ & $41(74.5)$ & $19(5 \mathrm{I} .4)$ & 0.039 & $2.88(1.11-7.45)$ \\
\hline No & $32(34.8)$ & $14(25.5)$ & I8 (48.6) & & \\
\hline
\end{tabular}

Note: *Adjusted for age and drug resistance to ofloxacin. 
test may represent a powerful tool for predicting treatment efficacy, thereby aiding clinical doctors in decisions related to changes in treatment protocols. Moreover, due to the increase in patients' failures to follow-up after 6 months of treatment, the ability to use a patient's bacteriology status at 2 months after treatment would provide a means of predicting that patient's treatment prognosis early in his/her treatment.

\section{Limitations}

There are several limitations in our study. Firstly, this study was performed in one city in China, such that one must be careful not to generalize the findings to other areas. Secondly, in patients for whom anti-TB therapy was prolonged, the regular sputum tests were frequently interrupted, providing insufficient data to evaluate the effectiveness of using longterm consecutive bacteriology conversions to predict TB treatment outcomes.

\section{Conclusion}

The prognosis of MDR-TB patients exhibiting persistent sputum positivity was inferior to those patients exhibiting sputum bacteriology conversion. Sputum smear conversions occurring at 2 months after treatment initiation may effectively predict treatment outcomes of MDR-TB patients.

\section{Acknowledgments}

This study was supported by the National Natural Science Foundation of China (81473027), the Qing Lan Project (2014), the Six Talent Peaks Project in Jiangsu Province (2014-YY-023), and the Priority Academic Program Development of Jiangsu Higher Education Institutions (PAPD). The funders had no role in the study design, data collection and analysis, decision to publish, or preparation of the manuscript.

\section{Author contributions}

All authors contributed toward data analysis, drafting and critically revising the paper and agree to be accountable for all aspects of the work.

\section{Disclosure}

The authors report no conflicts of interest in this work.

\section{References}

1. Dheda K, Barry CE 3rd, Maartens G. Tuberculosis. Lancet. 2016;387(10024):1211-1226.

2. Marais BJ. The global tuberculosis situation and the inexorable rise of drug-resistant disease. Adv Drug Deliv Rev. 2016;102:3-9.
3. World Health Organization. Global tuberculosis report 2015. 2016; Available from: http://www.who.int/tb/publications/2016/en/. Accessed Aug 8, 2017.

4. Zhang J, Gou H, Hu X, et al. Status of drug-resistant tuberculosis in China: a systematic review and meta-analysis. Am J Infect Control. 2016;44(6):671-676.

5. Shao Y, Yang D, Xu W, et al. Epidemiology of anti-tuberculosis drug resistance in a Chinese population: current situation and challenges ahead. BMC Public Health. 2011;11:110.

6. Pablos-Mendez A, Raviglione MC, Laszlo A, et al. Global surveillance for antituberculosis-drug resistance, 1994-1997. World Health Organization - International Union against Tuberculosis and Lung Disease Working Group on Anti-Tuberculosis Drug Resistance Surveillance. N Engl J Med. 1998;338(23):1641-1649.

7. Diacon AH, Pym A, Grobusch MP, et al. Multidrug-resistant tuberculosis and culture conversion with bedaquiline. $N$ Engl J Med. 2014;371(8):723-732.

8. World Health Organization. Treatment of tuberculosis: Guidelines for National Programmes. 2003. Available from: http://www.who.int/ docstore/gtb/publications/ttgnp/. Accessed Aug 8, 2017.

9. Lu P, Liu Q, Martinez L, et al. Time to sputum culture conversion and treatment outcome of patients with multidrug-resistant tuberculosis: a prospective cohort study from urban China. Eur Respir J. 2017;49(3):1601558.

10. World Health Organization. Guidelines for the programmatic management of drug-resistant tuberculosis: emergency update. 2008. Available at from: http://www.who.int/tb/challenges/mdr/programmatic_guidelines_for_mdrtb/en/. Accessed Aug 8, 2017.

11. [no authors listed]. Guidelines for surveillance of drug resistance in tuberculosis. WHO Geneva/IUATLD Paris. International Union against Tuberculosis and Lung Disease. Int J Tuberc Lung Dis. 1998;2(1): 72-89.

12. Kurbatova EV, Cegielski JP, Lienhardt C, et al. Sputum culture conversion as a prognostic marker for end-of-treatment outcome in patients with multidrug-resistant tuberculosis: a secondary analysis of data from two observational cohort studies. Lancet Respir Med. 2015;3(3):201-209.

13. Tupasi TE, Garfin AM, Kurbatova EV, et al. Factors associated with loss to follow-up during treatment for multidrug-resistant tuberculosis, the Philippines, 2012-2014. Emerg Infect Dis. 2016;22(3):491-502.

14. Kuaban C, Noeske J, Rieder HL, Ait-Khaled N, Abena Foe JL, Trebucq A. High effectiveness of a 12-month regimen for MDR-TB patients in Cameroon. Int J Tuberc Lung Dis. 2015;19(5):517-524.

15. Bastos ML, Lan Z, Menzies D. An updated systematic review and metaanalysis for treatment of multidrug-resistant tuberculosis. Eur Respir J. 2017;49(3):1600803.

16. Perrin FM, Lipman MC, McHugh TD, Gillespie SH. Biomarkers of treatment response in clinical trials of novel antituberculosis agents. Lancet Infect Dis. 2007;7(7):481-490.

17. Peloquin CA. Therapeutic drug monitoring in the treatment of tuberculosis. Drugs. 2002;62(15):2169-2183.

18. Holtz TH, Sternberg M, Kammerer S, et al. Time to sputum culture conversion in multidrug-resistant tuberculosis: Predictors and relationship to treatment outcome multidrug-resistant TB sputum culture conversion. Ann Intern Med. 2006;144(9):650-659.

19. Phillips PP, Davies GR, Mitchison DA. Biomarkers for tuberculosis disease activity, cure, and relapse. Lancet Infect Dis. 2010;10(2): 69-70.

20. Horne DJ, Royce SE, Gooze L, et al. Sputum monitoring during tuberculosis treatment for predicting outcome: systematic review and meta-analysis. Lancet Infect Dis. 2010;10(6):387-394.

21. Kurbatova E, Gammino VM, Bayona J, et al. Predictors of sputum culture conversion among patients treated for multidrug-resistant tuberculosis. Int J Tuberc Lung Dis. 2012;16(10):1335-1343. 


\section{Publish your work in this journal}

Infection and Drug Resistance is an international, peer-reviewed openaccess journal that focuses on the optimal treatment of infection (bacterial, fungal and viral) and the development and institution of preventive strategies to minimize the development and spread of resistance. The journal is specifically concerned with the epidemiology of antibiotic resistance and the mechanisms of resistance development and diffusion in both hospitals and the community. The manuscript management system is completely online and includes a very quick and fair peerreview system, which is all easy to use. Visit http://www.dovepress.com/ testimonials.php to read real quotes from published authors.

Submit your manuscript here: https://www.dovepress.com/infection-and-drug-resistance-journal 